\title{
The geoid of China and its surrounding areas determined by shallow layer method
}

\author{
Youchao Xie ${ }^{1,2,3}$, Wen-Bin Shen ${ }^{1,2,4, *}$, Jiancheng Han ${ }^{5}$, and Jiancheng $\operatorname{Li}^{1}$ \\ ${ }^{1}$ School of Geodesy and Geomatics, Wuhan University, Wuhan, China \\ ${ }^{2}$ Time and Frequency Geodesy Center, Wuhan University, Wuhan, China \\ ${ }^{3}$ Shanghai Municipal Engineering Design Institute (Group) Co., LTD, Shanghai, China \\ ${ }^{4}$ State Key Laboratory of Information Engineering in Surveying, Mapping and Remote Sensing, Wuhan University, Wuhan, China \\ ${ }^{5}$ Institute of Geophysics, China Earthquake Administration, Beijing, China
}

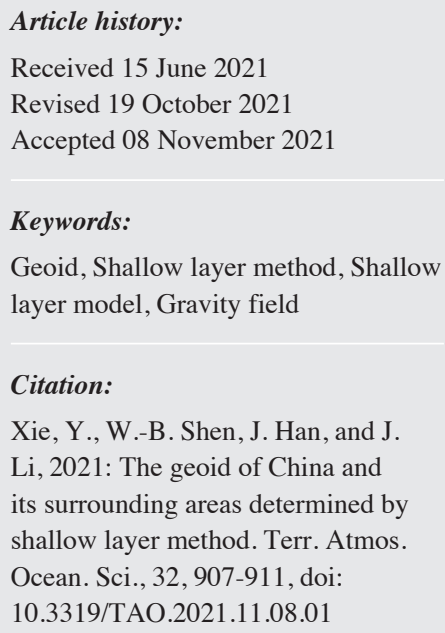

Citation:

Xie, Y., W.-B. Shen, J. Han, and J. Li, 2021: The geoid of China and its surrounding areas determined by shallow layer method. Terr. Atmos. Ocean. Sci., 32, 907-911, doi: 10.3319/TAO.2021.11.08.01

\begin{abstract}
In this study, we established a 5' $\times 5^{\prime}$ China geoid model 2021, which is determined based on the shallow layer method (SLM, Shen 2006). We use the external global Earth gravity field models (EGM2008 and EIGEN-6C4), the digital topographic model DTM2006.0 and crust model CRUST1.0 to construct the shallow layer model. The top and bottom boundaries of the shallow layer model are the Earth's nature surface and a surface below a reference geoid for a certain depth. Then the gravity field in the region inside the shallow layer can be determined. Based on the definition of the geoid $W=W_{0}$ (where $W_{0}=62636851.7 \mathrm{~m}^{2} \mathrm{~s}^{-2}$ is the geopotential constant on the geoid), we determined the geoid in and around China, which is referred to as CGM2021 (including CGM2021-08 and CGM2021-6C4). Comparisons show that the differences between the CGM2021 and EGM2008 geoid or EIGEN$6 \mathrm{C} 4$ geoid in China are in centimeter level in average.
\end{abstract}

\section{INTRODUCTION}

As the equi-geopotential surface that is closest to the mean sea surface, the geoid is of great significance. Firstly, the shape of the geoid really reflects the internal structure and density distribution of the earth. Secondly, the unification of global elevation datum is an urgent problem to be solved in geodesy due to the fact that there are differences among various regional height systems. The geoid, as the datum of the orthometric height, is the key to realize the unification of global elevation datum. Therefore, the determination of a high-precision global geoid is the key task in the field of geodesy. At present, the geoid determination methods mainly rely on two classical theories, Stokes' theory and Molodensky's theory, but these two theories have some kinds of limitations (Heiskanen and Moritz 1967; HofmannWellenhof and Moritz 2006).

In Stokes' theory (Stokes 1849), taking the geoid as

\footnotetext{
* Corresponding author

E-mail:wbshen@sgg.whu.edu.cn
}

boundary, one has to remove the masses outside the geoid and shift them into the inside of the geoid, namely the masses outside the geoid should be adjusted, which gives rise to the variation of the geoid (Helmert 1884; Lambert 1930; Vaníček and Martinec 1994; Vaníček et al. 1995; Martinec 1998). In another aspect, to make mass adjustment, we need to know a priori orthometric height $\mathrm{H}$ above the geoid, but the latter is to be determined (Bruns 1878). Hence, an iteration procedure is needed to finally determine the geoid.

In order to avoid the limitations of Stokes' method, Molodensky used the Earth's surface as boundary (Molodensky et al. 1962; Heiskanen and Moritz 1967; Hofmann-Wellenhof and Moritz 2006). According to Molodensky's theory, a quasi-geoid rather than geoid is determined. Though Molodensky's theory does not need to make mass adjustment, the quasi-geoid determined is not an equi-geopotential surface.

To overcome the above mentioned drawbacks in Stokes' theory and Molodensky's theory, Shen (2006) proposed the shallow layer method (SLM). The basic idea is stated as 
follows. Generally, the geoid lies beneath the Earth's natural surface of the continents. The geopotential on the geoid cannot be directly obtained by observation. Shen (2006) proposed the SLM and constructed a shallow layer model to solve this problem. An external global Earth gravity field model (EGM2008 and EIGEN-6C4 models) is used as input information, the digital topographic model DTM2006.0, the DNSC08 mean sea surface (MSS) and the crust model CRUST1.0 (Pavlis et al. 2008, 2012; Andersen and Knudsen 2009; Andersen et al. 2010; Laske et al. 2013; Förste et al. 2014) are used to construct the shallow layer model. The top and bottom boundaries of the shallow layer model are the Earth's natural surface and a surface below a reference geoid for a certain depth (see Fig. 1) (Han 2012; Shen 2013).

Then (refering to Fig. 1), the gravitational potential generated by the whole Earth can be divided into two parts. One part is the gravitational potential generated by the inner mass that is enclosed by $\Gamma$, and another part is the gravitational potential generated by the shallow layer mass. We can calculate the gravitational potential generated by the shallow layer mass by Newtonian integral, expressed in spherical harmonic coefficients outside the Earth's surface $S$. Then using the global gravitational field model (e.g., EGM2008 or EIGEN-6C4), which is generated by the whole mass of the Earth, subtracting the gravitational potential generated by the shallow layer mass, we obtain the gravitational potential generated by the inner mass in the domain outside $S$. Then through natural continuation, we can obtain the spherical harmonic coefficients of the gravitational potential generated by the inner mass outside $\Gamma$. The so-called natural continuation is that the spherical harmonic coefficients of the field generated by inner mass outside Earth's surface $S$ is consistent with the spherical harmonic coefficients of the field outside the $\Gamma$. Suppose we have two regular harmonic functions, both of which are defined in the domain outside $\Gamma$. If they are consistent in the domain outside Earth's surface $S$, then they must be consistent in the domain outside $\Gamma$. And then the geopotential of any point outside $\Gamma$ can be obtained. Based on the equation, $W=W_{0}$ defining the geoid, we may determine points that satisfy this equation. These points construct the geoid. For details refer to Shen (2006) and Shen and Han (2013).

The advantage of the SLM is that it is based on the definition of the geoid, thereby avoiding boundary-value problems and mass adjustment. Shen and Han (2013) showed that the results of the determined geoid undulation based on the SLM are better than those determined by other methods. As a reference, orthometric heights derived from the GPS leveling data in Xinjiang, China were used.

\section{THE CHINESE GEOID MODEL CGM2021}

Using SLM and based on two different gravity field models EGM2008 and EIGEN-6C4, and the density model CRUST1.0, we calculated respectively the Chinese geoid models CGM2021-08 and CGM2021-6C4 (generally referred to as CGM2021), including China and a rectangle area around China, as shown in Fig. 2. The statistics information is listed in Table 1.

As we can see from Table 1, the maximum, minimum, mean, and standard deviation of the Chinese geoid calculated by SLM and based on EGM2008 are respectively 46.766 , $-75.613,-21.896,30.561 \mathrm{~m}$ and those of the EGM2008 geoid in China (calculated based on a conventional approach as provided by ICGEM: http://icgem.gfz-potsdam.de/home, Ince et al. 2019) are respectively $46.648,-75.399,-21.946$, $30.510 \mathrm{~m}$. The maximum, minimum, mean, and standard deviation of the China geoid calculated by SLM and based on EIGEN-6C4 are respectively 46.749, -75.656, -21.897, $30.563 \mathrm{~m}$ and those of the EIGEN-6C4 geoid in China (calculated based on conventional approach as provided by ICGEM) are respectively $46.637,-75.420,-21.945,30.513 \mathrm{~m}$. The EGM2008 geoid and EIGEN-6C4 geoid in China are almost the same and the geoid calculated by the shallow layer method is very close to EGM2008 geoid or EIGEN6C4 geoid in China.

\section{THE COMPARATION BETWEEN CGM2021 AND EGM2008/EIGEN-6C4 GEOID IN CHINA}

As evaluation, the CGM2021 (including CGM2021-08 and CGM2021-6C4) is compared to the EGM 2008 geoid and EIGEN-6C4 geoid in China. Grid points with a grid distance of one degree in longitude and latitude, in total 2405 points, are for comparison (see Fig. 3).

It is shown in Table 2 that the differences of geoid between SLM and the corresponding independent gravitational field model are very small for 2405 points in China and its surrounding areas. The maximum, minimum, mean, standard deviation, and root mean square of the difference between CGM2021-08 and EGM2008 geoid are 0.186, $-0.853,-0.040,0.050,0.064 \mathrm{~m}$ respectively. The maximum, minimum, mean, standard deviation, and root mean square of the difference between CGM2021-6C4 and EIGEN-6C4 geoid are $0.187,-0.851,-0.039,0.050,0.063 \mathrm{~m}$ respectively. In both cases, the mean, standard deviation, and root mean square of the differences are very small. This indicates that the geoid calculated based on SLM is very close to the well-known independent gravitational field models in this region. But we can see that the differences between the results of different gravitational field models are larger. Figure 4 shows that several regions with large differences are mainly distributed in the Qinghai-Tibet Plateau and Xinjiang region and other areas with complex terrain and the values of Figs. $4 \mathrm{~b}$ and $\mathrm{d}$ are inversely distributed compared to Figs. 4a and c. 


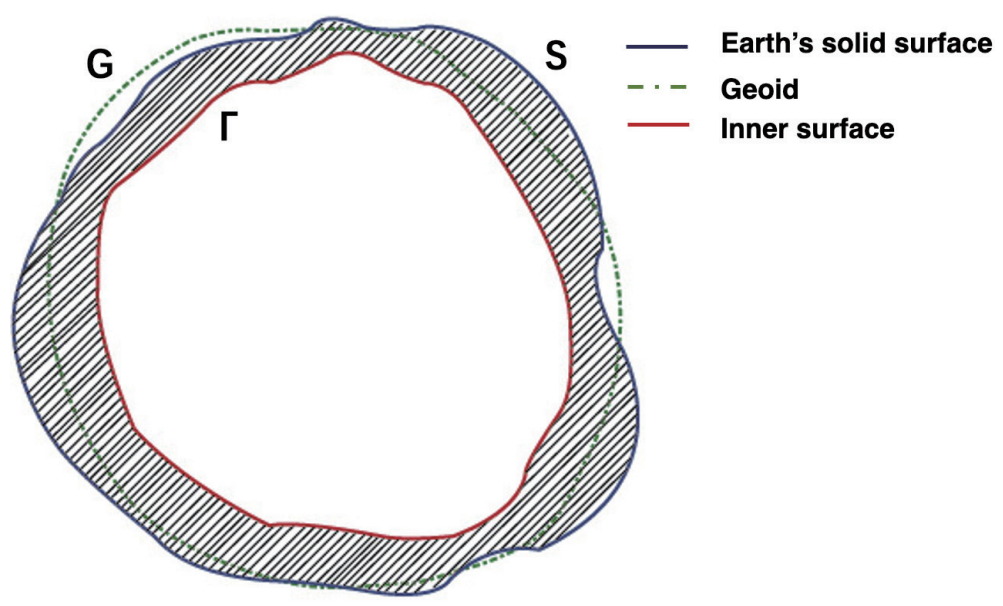

Fig. 1. Definition of the shallow layer, redrawn after Shen and Han (2013). The closed solid blue curve denotes the Earth's surface $S$; the closed dotted green curve denotes the geoid $G$; and the solid red curve denotes an inner surface $\Gamma$ below the geoid. The mass volume bounded by $\Gamma$ and $S$ is referred to as the shallow mass layer.

(a)

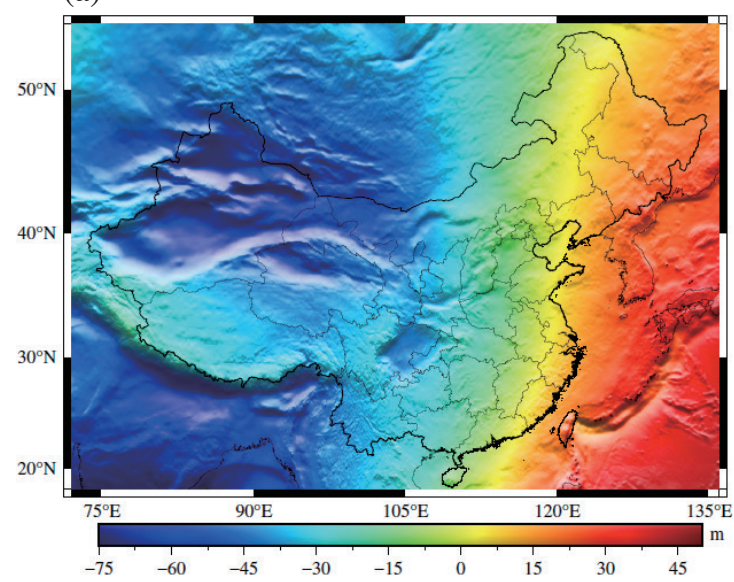

(b)

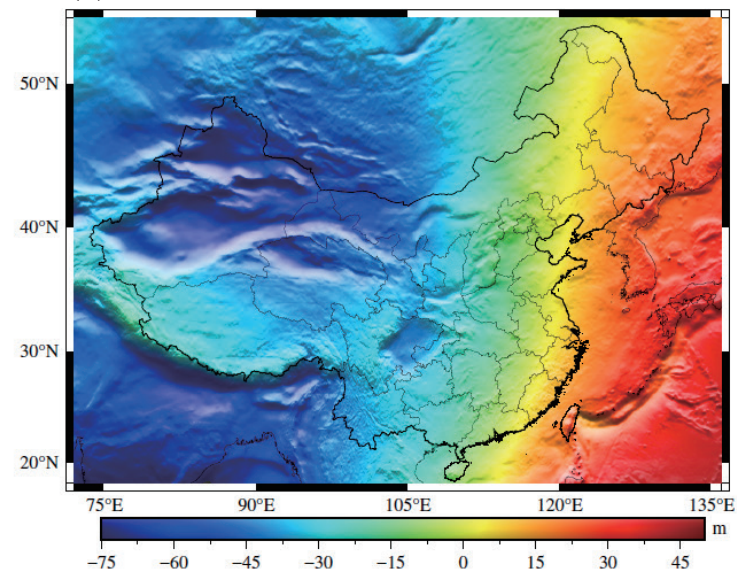

Fig. 2. The calculated 5' × 5' geoid model CGM2021. (a) CGM2021-08 based on EGM2008; (b) CGM2021-6C4 based on EIGEN-6C4.

Table 1. The statistics of the two geoid models of China CGM202108 and CGM2021-6C4, both determined by the shallow layrer method (SLM) and based on EGM2008 and EIGEN-6C4 respectively (unit: $\mathrm{m}$ ).

\begin{tabular}{c|cccc}
\hline Model & Max & Min & Mean & STD \\
\hline CGM2021-08 & 46.766 & -75.613 & -21.896 & 30.561 \\
EGM2008 geoid & 46.648 & -75.399 & -21.946 & 30.510 \\
CGM2021-6C4 & 46.749 & -75.656 & -21.897 & 30.563 \\
EIGEN-6C4 geoid & 46.637 & -75.420 & -21.945 & 30.513 \\
\hline
\end{tabular}




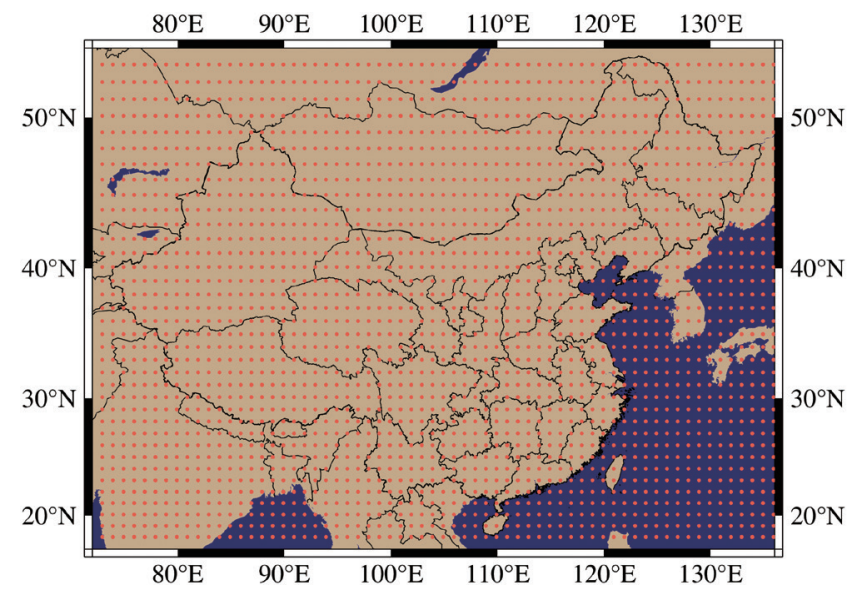

Fig. 3. The 2405 discrete points for comparation, and the node interval is 1 degree.

Table 2. The difference between the geoids of China (CGM2021-08 and CGM2021-6C4) determined by SLM and EGM2008 geoid and EIGEN-6C4 geoid (unit: m).

\begin{tabular}{c|ccccc}
\hline Model & Max & Min & Mean & STD & RMS \\
\hline CGM2021-08 - EGM2008 geoid & 0.186 & -0.853 & -0.040 & 0.050 & 0.064 \\
CGM2021-08 - EIGEN-6C4 geoid & 1.981 & -3.181 & -0.041 & 0.2512 & 0.255 \\
CGM2021-6C4 - EIGEN-6C4 geoid & 0.187 & -0.851 & -0.039 & 0.050 & 0.063 \\
CGM2021-6C4 - EGM2008 geoid & 3.025 & -2.195 & -0.040 & 0.235 & 0.238 \\
\hline
\end{tabular}

(a)

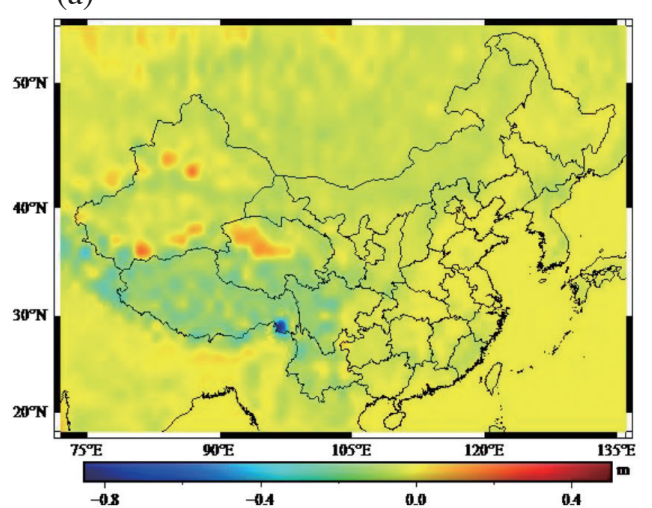

(c)

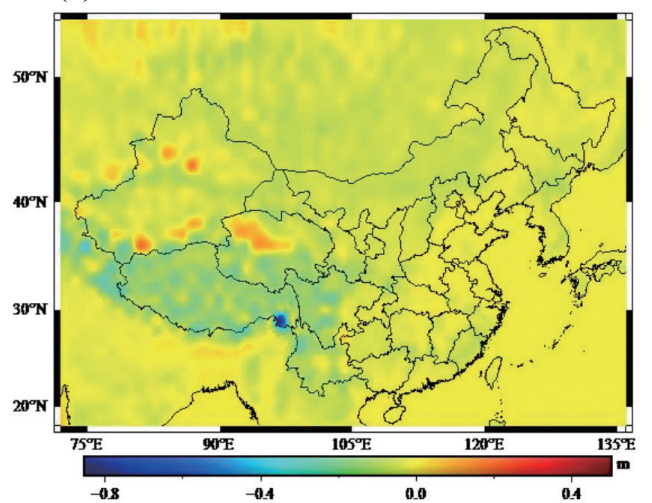

(b)

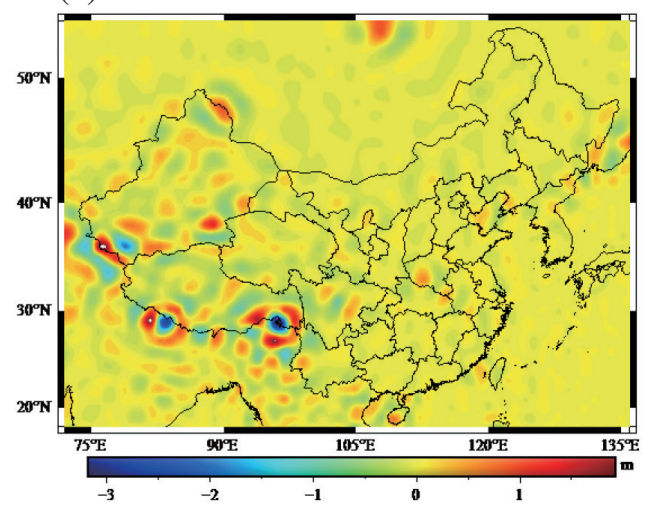

(d)

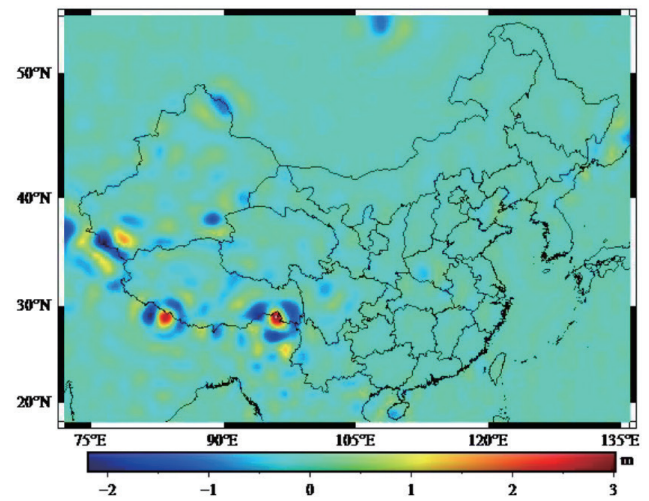

Fig. 4. The difference between (a) CGM2021-08 and EGM2008 geoid, (b) CGM2021-08 and EIGEN-6C4 geoid, (c) CGM2021-6C4 and EIGEN6C4 geoid, and (d) CGM2021-6C4 and EGM2008 geoid. 


\section{CONCLUSIONS}

In this study, we established the China geoid model CGM2021 for China and its surrounding areas by using SLM. If the external gravity field model EGM2008/ EIGEN-6C4 is applied, the China geoid model CGM202108/CGM2021-6C4 is established. We selected 2405 points in the region, and compared the differences between the CGM2021 and the EGM2008/EIGEN-6C4 geoid at these points. The results show that the CGM2021 based on SLM is very close to the EGM2008/EIGEN-6C4 geoid, and the average difference between CGM2021 and EGM2008/EIGEN-6C4 geoid in China is in centimeter level. This verifies the reliability and the accuracy of the shallow layer method.

Acknowledgements We would like to express our sincere thanks to C. Hwang and two anonymous reviewers for their valuable comments and suggestions, which greatly improved the manuscript. This work is supported by the National Natural Science Foundations of China (Nos. 42030105, 41721003, 41804012, 41631072, 41874023), Space Station Project (2020) (No. 228), and the Natural Science Foundation of Hubei Province of China (No. 2019CFB611).

\section{REFERENCES}

Andersen, O. B. and P. Knudsen, 2009: Dnsc08 mean sea surface and mean dynamic topography models. J. Geophys. Res., 114, C11001, doi: 10.1029/2008JC005179. [Link]

Andersen, O. B., P. Knudsen, and P. A. M. Berry, 2010: The DNSC08GRA global marine gravity field from double retracked satellite altimetry. J. Geod., 84, 191-199, doi: 10.1007/s00190-009-0355-9. [Link]

Bruns, H., 1878: Die Figur der Erde: Ein Beitrag zur europäischen Gradmessung, Publ. Preuss. Geod. Inst., Berlin, 49 pp.

Förste, C., S. L. Bruinsma, O. Abrykosov, J.-M. Lemoine, J. C. Marty, F. Flechtner, G. Balmino, F. Barthelmes, and R. Biancale, 2014: EIGEN-6C4 The latest combined global gravity field model including GOCE data up to degree and order 2190 of GFZ Potsdam and GRGS Toulouse. GFZ Data Services, doi: 10.5880/icgem.2015.1. [Link]

Han, J., 2012: Geoid determination based on the Earth gravity model and the gravity potential of the shallow Layer. Ph.D. Thesis, Wuhan University, Wuhan. (in Chinese)

Heiskanen, W. A. and H. Moritz, 1967: Physical Geodesy, W. H. Freeman and Company, San Francisco, 364 pp.

Helmert, F. R., 1884: Die Mathematischen Und Physikalischen Theorien Der Höheren Geodäsie, B. G. Teubner, Leipzig.

Hofmann-Wellenhof, B. and H. Moritz, 2006: Physical
Geodesy, 2nd Edition, Springer, Vienna, 403 pp, doi: 10.1007/978-3-211-33545-1. [Link]

Ince, E. S., F. Barthelmes, S. Reißland, K. Elger, C. Förste, F. Flechtner, and H. Schuh, 2019: ICGEM - 15 years of successful collection and distribution of global gravitational models, associated services, and future plans. Earth System Science Data, 11, 647-674, doi: 10.5194/ essd-11-647-2019. [Link]

Lambert, W. O., 1930: The reduction of observed values of gravity to sea level. Bull. Géodésique, 26, 107-181, doi: 10.1007/bf03030029. [Link]

Laske, G., G. Masters, Z. Ma, and M. Pasyanos, 2013: Update on CRUST1.0 - A 1-degree global model of earth's crust. Geophysical Research Abstracts, Vol. 15, EGU2013-2658, EGU General Assembly 2013, Vienna, Austria.

Martinec, Z., 1998: Boundary-Value Problems for Gravimetric Determination of a Precise Geoid, Springer, Berlin, Heidelberg, 228 pp, doi: 10.1007/BFb0010337. [Link]

Molodensky, M. S., V.F. Eremeev, and M. I. Yurkina, 1962: Methods for Study of the External Gravitational Field and Figure of the Earth (transl. from Russian 1960), Israel Program for Scientific Translations, Jerusalem.

Pavlis, N. K., S. A. Holmes, S. C. Kenyon, and J. K. Factor, 2008: An earth gravitational model to degree 2160: Egm2008. EGU General Assembly 2008, Vienna, Austria.

Pavlis, N. K., S. A. Holmes, S. C. Kenyon, and J. K. Factor, 2012: The development and evaluation of the Earth Gravitational Model 2008 (EGM2008). J. Geophys. Res., 117, doi: 10.1029/2011JB008916. [Link]

Shen, W.-B., 2006: An approach for determining the precise global geoid. 1st International Symposium of the International Gravity Field Service, Istanbul, Turkey.

Shen, W.-B., 2013: A new formulation of stokes' approach in determining the global gravimetric geoid. Ann. Geophys., 56, R0325, doi: 10.4401/ag-5749. [Link]

Shen, W.-B. and J. Han, 2013: Improved geoid determination based on the shallow-layer method: A case study using EGM08 and CRUST2.0 in the Xinjiang and Tibetan regions. Terr. Atmos. Ocean. Sci., 24, 591-604, doi: 10.3319/TAO.2012.11.12.01(TibXS). [Link]

Stokes, G. G., 1849: On the variation of gravity at the surface of the earth. Trans. Camb. Phil. Soc., 8, 672-695.

Vaníček, P. and Z. Martinec, 1994: The Stokes-Helmert scheme for the evaluation of a precise geoid. Manuscr Geod., 19, 119-128.

Vaníček, P., M. Najafi,Z. Martinec, L. Harrie, and L. E. Sjöberg, 1995: Higher-degree reference field in the generalized Stokes-Helmert scheme for geoid computation. J. Geod., 70, 176-182, doi: 10.1007/bf00943693. [Link] 